\title{
Microglial Activation in Perinatal Rabbit Brain Induced by Intrauterine Inflammation: Detection with ${ }^{11} \mathrm{C}-(R)-\mathrm{PK} 11195$ and Small-Animal PET
}

\author{
Sujatha Kannan ${ }^{1}$, Fadoua Saadani-Makki ${ }^{1}$, Otto Muzik ${ }^{1,2}$, Pulak Chakraborty ${ }^{2}$, Thomas J. Mangner ${ }^{2}$, James Janisse ${ }^{3}$, \\ Roberto Romero ${ }^{4,5}$, and Diane C. Chugani ${ }^{1,2}$ \\ ${ }^{1}$ Carman and Ann Adams Department of Pediatrics, Wayne State University, Detroit, Michigan; ${ }^{2}$ Department of Radiology, Wayne State \\ University School of Medicine, Detroit, Michigan; ${ }^{3}$ Department of Medicine, Wayne State University School of Medicine, Detroit, \\ Michigan; ${ }^{4}$ Perinatology Research Branch, NICHD, National Institutes of Health, Department of Health and Human Services, Bethesda, \\ Maryland and Detroit, Michigan; and ${ }^{5}$ Center for Molecular Medicine and Genetics, Wayne State University School of Medicine, \\ Detroit, Michigan
}

Intrauterine infection can lead to a fetal inflammatory response syndrome that has been implicated as one of the causes of perinatal brain injury leading to periventricular leukomalacia (PVL) and cerebral palsy. The presence of activated microglial cells has been noted in autopsy specimens of patients with PVL and in models of neonatal hypoxia and ischemia. Activated microglial cells can cause oligodendrocyte damage and white matter injury by release of inflammatory cytokines and production of excitotoxic metabolites. We hypothesized that exposure to endotoxin in utero leads to microglial activation in the fetal brain that can be monitored in vivo by ${ }^{11} \mathrm{C}-(R)-\mathrm{PK} 11195$ (1-[2-chlorophenyl]$N$-methyl- $N$-[1-methylpropyl]-3-isoquinoline carboxamide) - a positron-emitting ligand that binds peripheral benzodiazepine receptor sites in activated microglia-using small-animal PET. Methods: Pregnant New Zealand White rabbits underwent laparotomy and were injected with 20 and $30 \mu \mathrm{g} / \mathrm{kg}$ of Escherichia coli lipopolysaccharide along the length of the uterus on day 28 of gestation. The pups were born spontaneously at term (31 d) and were scanned using small-animal PET after intravenous administration of ${ }^{11} \mathrm{C}-(R)-\mathrm{PK} 11195$ and by MRI on postnatal day 1. The standard uptake values (SUVs) of the tracer were calculated for the whole brain at 10-min intervals for 60 min after tracer injection. The pups were euthanized after the scan, and brains were fixed, sectioned, and stained for microglial cells using biotinylated tomato lectin. Results: There was increased brain retention of ${ }^{11} \mathrm{C}-(R)-\mathrm{PK} 11195$ - as determined by a significant difference in the slope of the SUV over time-in the endotoxintreated pups when compared with that of age-matched controls. Immunohistochemical staining showed dose-dependent changes in activated microglia (increased number and morphologic changes) in the periventricular region and hippocampus of the brain of newborn rabbit pups exposed to endotoxin in utero. Conclusion: Intrauterine inflammation leads to activation of microglial cells that may be responsible for the development of brain injury and white matter damage in the perinatal period.

Received Dec. 22, 2006; revision accepted Mar. 16, 2007.

For correspondence or reprints contact: Sujatha Kannan, MD, Carman and Ann Adams Department of Pediatrics, Children's Hospital of Michigan, Wayne State University, 3901 Beaubien Blvd., Detroit, MI 48201.

E-mail: skannan@med.wayne.edu

COPYRIGHT @ 2007 by the Society of Nuclear Medicine, Inc.
PET with the tracer ${ }^{11} \mathrm{C}-(R)-\mathrm{PK} 11195$ can be used as a noninvasive, sensitive tool for determining the presence and progress of neuroinflammation due to perinatal insults in newborns.

Key Words: perinatal brain injury; intrauterine inflammation; small-animal PET; ${ }^{11} \mathrm{C}-(R)$-PK11195; microglia

J Nucl Med 2007; 48:946-954

DOI: 10.2967/jnumed.106.038539

$\mathbf{I}$ ntrauterine infection is known to be a risk factor for brain injury in the perinatal period. Several clinical studies have also noted the significant correlation between intrauterine infection, perinatal brain injury, periventricular leukomalacia (PVL), and cerebral palsy (1-3). Intrauterine infection leads to a fetal systemic inflammatory response mediated by cytokines that has been implicated in the development of PVL and neuronal damage in the infant. PVL is frequently associated with severe neurologic disorders, such as cerebral palsy and cognitive impairment, which lead to lifelong disabilities in children.

In recent years several in vitro and in vivo studies have implicated microglial cells in the development of PVL in hypoxic-ischemic brain injuries. Haynes et al. have shown the increased presence of activated microglia diffusely throughout the white matter in autopsy specimens of patients with PVL, indicating that activated microglia are involved in causing white matter damage by oxidative and nitrosative stress (4). Proinflammatory cytokines, such as tumor necrosis factor- $\alpha$ and interleukin- $1 \beta$, have been shown to induce microglial activation in vitro $(5,6)$. Oligodendrocyte damage with lipopolysaccharide (LPS) treatment appears to occur only in the presence of microglial cells, supporting the role of microglial cell activation in white matter injury (7). There are several mechanisms by which activated microglia may induce white matter injury. When activated, microglial cells have been shown to release reactive nitrogen 
and reactive oxygen species into the surrounding areas, causing damage to oligodendrocytes (8). Activated microglia are also known to release excitotoxic metabolites, such as glutamate (5) and quinolinic acid (9), which may cause glutamate receptor- or $N$-methyl-D-aspartic acid receptormediated injury to oligodendrocytes. These studies indicate that activation of microglial cells may play a role in the development of PVL and white matter injury in infants exposed to intrauterine infections.

PK11195 (1-[2-chlorophenyl]- $N$-methyl- $N$-[1-methylpropyl]3 -isoquinoline carboxamide) is an antagonist that is specific for the peripheral benzodiazepine receptor (PBR) binding sites localized on the outer mitochondrial membrane of activated microglia and macrophages (10). Peripheral benzodiazepine binding sites are not significantly expressed in normal brain parenchyma but increased binding of PK11195 is found predominantly on activated microglia secondary to neuronal damage $(10)$. The $(R)$ enantiomer has been shown to have higher affinity for the peripheral benzodiazepine binding sites than the $(S)$ enantiomer $(11)$; therefore, ${ }^{11} \mathrm{C}-(R)-\mathrm{PK} 11195$ was used in these studies. When labeled with ${ }^{11} \mathrm{C},(R)-\mathrm{PK} 11195$ can be used effectively as a ligand for PET studies, indicating the presence of activated microglia in neuroinflammatory diseases $(12,13)$, neurodegenerative disorders $(14,15)$, and stroke $(16,17)$. We hypothesized that exposure to endotoxin in utero would lead to microglial activation that can be demonstrated in vivo in real time, noninvasively by PET using the $\operatorname{tracer}{ }^{11} \mathrm{C}-(R)-\mathrm{PK} 11195$.

\section{MATERIALS AND METHODS}

\section{Animal Model}

All animal procedures were approved by the Animal Investigation Committee of Wayne State University. New Zealand White rabbits were chosen for this study because of the similarity in their brain development to that of human brain myelination. Pregnant New Zealand White rabbits with timed pregnancies, that were confirmed breeders with a history of delivering 7-10 kits per litter, were purchased from Covance Inc. A laparotomy was done under general anesthesia on gestational day 28 (term pregnancy is 31-32 d), and $1 \mathrm{~mL}$ of saline for the control group $(n=4)$ or $1 \mathrm{~mL}$ of saline containing $20(n=4), 30(n=3)$, or $40(n=3) \mu \mathrm{g} / \mathrm{kg}$ of Escherichia coli O127:B8 (Sigma) LPS for the endotoxin groups was equally divided and injected using a 26-gauge needle between the fetuses along the length of the uterine wall, taking care not to enter the amniotic sac. This ensured that all kits were exposed to the same amount of endotoxin. The peritoneum and abdominal wall were closed in layers. The dams were monitored daily for changes in activity, feeding, and fever. The kits were all born spontaneously at 31-d gestational age, and the litter size ranged from 5 to 11 kits. The number of live and dead kits was recorded and dead kits were removed from the cage. One or 2 kits from each litter were randomly picked for PET, which was followed by MRI for coregistration of the PET images.

\section{Radiosynthesis and Formulation of ${ }^{11} \mathrm{C}-(\boldsymbol{R})-\mathrm{PK} 11195$}

The ${ }^{11} \mathrm{C}-(R)-\mathrm{PK} 11195$ was synthesized using a procedure described by Chakraborty et al. (18), in which the amide nitrogen of the precursor, $(R)-N$-desmethyl PK11195, was methylated with
${ }^{11} \mathrm{C}$-methyl iodide. The labeled product was purified by semipreparative high-performance liquid chromatography on a C-18 column using $35 \%$ aqueous ethanol as eluent. The pure product was collected in a vial containing Tween-80 (Sigma) and diluted with normal saline solution (10-12 mL) before sterilization through a sterile $0.2-\mu \mathrm{m}$ nylon filter. The Tween-80 (final concentration, $1.5 \%[\mathrm{v} / \mathrm{v}])$ was required in the formulation as a significant percentage of the product, as ${ }^{11} \mathrm{C}-(R)$-PK11195 is lost by adsorption onto the plastic material of sterile syringes and extension tubing if only aqueous ethanol is used in the formulation. Radiochemical and chemical purity of the product was $>98 \%$. The specific radioactivity of ${ }^{11} \mathrm{C}-(R)-\mathrm{PK} 11195$ was $0.0407 \pm$ $0.0074 \mathrm{MBq}$ at the end of synthesis, and at the time of administration the specific radioactivity was $>0.0148 \mathrm{MBq} / \mathrm{mmol}$.

\section{PET and MRI Protocols}

PET scans were performed using a microPET R4 tomograph (Siemens Preclinical Solutions). Because animal handling has considerable impact on the quality of PET images (19), the rabbit kits were kept warm during both preparation and imaging using a heating pad set at $37^{\circ} \mathrm{C}$. To achieve reproducible positioning of the rabbit kits in the microPET and MR scanner gantry, a head-andbody holder that was developed in house was used. The holder consists of a short plate on which the animal's body rests and is secured with multiple VELCRO straps (Velcro Industries) as well as with soft head-positioning knobs. Attached to the holder are 3 fixed spheres of 5-mm diameter, which are filled with small amounts of radioactivity before microPET imaging (fiducial markers). These spheres are visible on both the microPET (radioactivity) and the MR (water) images and are used for coregistration of the 2 imaging modalities. Using the head holder, the rabbit kits were placed in prone position on the microPET bed with the head in the center of the field of view. The kits were anesthetized with $30 \mathrm{mg} / \mathrm{kg}$ of ketamine and positioned on the head holder, and respirations, heart rate, and oxygen saturation were monitored during the scan. After an initial 17-min transmission scan to correct for attenuation of the 511-keV photons, the kits were injected intravenously in a peripheral vein with $10-20 \mathrm{MBq}$ of ${ }^{11} \mathrm{C}-(R)-\mathrm{PK} 11195$ (half-life, $20 \mathrm{~min}$ ), and a 60-min list-mode data acquisition was initiated. All scans were started at approximately 5 min after tracer injection. List-mode data were acquired in 3-dimensional (3D) mode and the coincidence data were subsequently rebinned into discrete time frames $(6 \times 10 \mathrm{~min})$ for kinetic analysis. The whole dynamic frame sequence was then reconstructed using attenuation correction and the ordered-subset expectation maximization (OSEM) iterative algorithm, yielding an isotropic resolution of about 2-mm full width at half maximization. After completion of the PET data acquisition, each animal underwent MRI for coregistration. MRI scans were performed using a 4.7-T Brucker magnet using a head coil with the animal in the head holder. High-resolution anatomic image volumes were acquired with a T1-weighted fast gradient-echo sequence with echo time $=8.4 \mathrm{~ms}$, repetition time $=20 \mathrm{~ms}, 8$ averages, and $20^{\circ}$ flip angle. A slab of 64 contiguous 0.5 -mm-thick slices was acquired in the coronal plane with a pixel size of $0.156 \times 0.156$ $\mathrm{mm}^{2}$. The total scanning time for this $3 \mathrm{D}$ acquisition was approximately $33 \mathrm{~min}$.

The images were subsequently processed using ASIPro microPET data analysis software (Siemens Preclinical Solutions). The MR and microPET image volumes were coregistered using the MPItool software (20) by matching the position of the 3 fiducial 
markers in both datasets. After coregistration, a 3D region of interest (ROI) involving the whole brain (cerebrum and midbrain up to the brain stem) was defined in the MR image volumes and copied to the dynamic image sequences, yielding dynamic timeactivity curves for the control and endotoxin groups. Because of partial-volume effects noted with microPET, multiple ROIs involving different areas of the brain were not analyzed due to the small size of the brain. The standardized uptake value (SUV) was calculated for each time point by dividing the mean tracer concentration $\left(\mathrm{MBq} / \mathrm{cm}^{3}\right)$ by the injected activity $(\mathrm{MBq})$ per weight $(\mathrm{g})$.

\section{Immunohistochemistry}

After PET and MRI scanning, the rabbit pups were perfused, under deep anesthesia, with phosphate-buffered saline (PBS, $\mathrm{pH}$ 7.4), which was followed by $4 \%$ paraformaldehyde. The brains were removed, immersed in the same fixative for $48 \mathrm{~h}$, cryoprotected using graded solutions of sucrose, and frozen at $80^{\circ} \mathrm{C}$ until they were sectioned. Coronal brain sections (30- $\mu \mathrm{m}$ thick) using a Leica cryostat were cut and mounted on slides coated with poly-Llysine (Sigma).

For immunohistochemical staining, the slides were washed with PBS, which was followed by incubation in $1 \%$ hydrogen peroxide for $30 \mathrm{~min}$ to inactivate the endogenous peroxidase, and then incubated in PBS solution containing 0.3\% Triton X-100 and $0.5 \%$ bovine serum albumin for $1 \mathrm{~h}$. The slides were covered with biotinylated Lycopersicon esculentum tomato lectin (1:100) (Vector Laboratories) for $1 \mathrm{~h}$. Avidin binding was performed using a Vectastain $\mathrm{ABC}$ kit (following instructions of the manufacturer) and developed using 3,3'-diaminobenzidine as a peroxidase substrate (Vector Laboratories). Slides were then rinsed in PBS, dehydrated in graded ethanol, cleared in xylene, mounted with mounting medium, and viewed using a Leica DM2500 microscope equipped with a camera.

\section{Statistical Analysis}

For the analysis of survival and weight, the kits are nested within the litters. When this type of nesting occurs, the unit of analysis should be the unit of randomization, which is the dam. The statistical techniques that are used should take the lack of independence into account. Hierarchical linear modeling (HLM) is one technique that properly models the lack of independence. To compare the weight of the kits across the 4 treatment groups, a 2-level HLM was used. In the first level of the model, the average weight of the kits within each litter was estimated. In the second level of the model, group differences were assessed. Because there were no deaths at birth and at $8 \mathrm{~h}$ in the control group, we were not able to run HLM with a Bernoulli outcome. To compare the survival rates, we obtained average survival rates within each litter and examined the monotonic decrease in survival across the 4 treatment groups with the Jonckheere test (21). For the analyses of SUV ( ${ }^{11}$ C-PK11195 uptake adjusted for weight of the kits and dose injected) across time, we had repeated measures nested within kits nested in litters. Because the kits from the $40-\mu \mathrm{g} / \mathrm{kg}$ endotoxin group died either during PET scanning or just before the scanning, these data were not included for the SUV analyses, but immunohistology was performed on these brain sections. To compare the change in SUV across time for the 3 groups (control as well as $20-$ and $30-\mu \mathrm{g} / \mathrm{kg}$ endotoxin groups), a 3-level HLM was used. In the first level of the model, slopes and intercepts for each kit were estimated. In the second level, average slopes and intercepts for the litters were estimated. In the third level, group differences were assessed. A $P$ value $<0.05$ was considered significant.

\section{RESULTS}

There were 35 kits from 4 litters in the control group, 33 kits from 4 litters in the 20- and $30-\mu \mathrm{g} / \mathrm{kg}$ endotoxin groups, and 31 kits from 3 litters in the $40-\mu \mathrm{g} / \mathrm{kg}$ endotoxin group. There was a significant monotonic decrease in the proportion of surviving kits at birth with an increase in the endotoxin dose $(P=0.008$; Kendall's $\tau=-0.59)$. The proportion (mean $\pm \mathrm{SD}$ ) of surviving kits was highest in the control group and decreased in groups treated with 20 , 30 , and $40 \mu \mathrm{g} / \mathrm{kg}$ of endotoxin $(1 \pm 0,0.88 \pm 0.40,0.82 \pm$ 0.47 , and $0.48 \pm 0.85$, respectively). There was no significant decrease in the weight of the kits with increasing doses of endotoxin $(P=0.093)$, but a greater difference was noted between the weight of the rabbit kits in the control group versus that in the $40-\mu \mathrm{g} / \mathrm{kg}$ endotoxin group (mean weight $\pm \mathrm{SD}$ was $42.6 \pm 11.44 \mathrm{~g}$ for the control group vs. $35.98 \pm 6.5 \mathrm{~g}$ for the $20-\mu \mathrm{g} / \mathrm{kg}, 36.96 \pm 5.52 \mathrm{~g}$ for the $30-\mu \mathrm{g} / \mathrm{kg}$, and $34.04 \pm 7.7 \mathrm{~g}$ for the $40-\mu \mathrm{g} / \mathrm{kg}$ endotoxin groups). A total of 4 kits from 3 different litters in the control and $30-\mu \mathrm{g} / \mathrm{kg}$ endotoxin groups and 4 kits from 4 different litters in the $20-\mu \mathrm{g} / \mathrm{kg}$ endotoxin group underwent PET and MRI and immunohistochemical analyses.

\section{PET and MRI}

A representative coregistration of the PET and MR images on day 1 of life for a rabbit kit exposed to $30 \mu \mathrm{g} / \mathrm{kg}$ of endotoxin in utero is shown in Figure 1. The average SUV of ${ }^{11} \mathrm{C}-(R)-\mathrm{PK} 11195$ for the whole brain and the predicted changes over time from the HLM analysis are shown in Figure 2. In the control group, ${ }^{11} \mathrm{C}-(R)-\mathrm{PK} 11195$ activity in the brain decreased with time (mean SUV slope $\pm \mathrm{SE}$ was $-0.003 \pm 0.0004 \mathrm{~min}^{-1}$ ), whereas the activity for the 20- and 30- $\mu \mathrm{g} / \mathrm{kg}$ endotoxin groups increased over time (mean SUV slopes with SE were $+0.0006 \pm 0.0004$ $\min ^{-1}$ and $+0.002 \pm 0.0004 \mathrm{~min}^{-1}$ for the $20-$ and $30-\mu \mathrm{g} / \mathrm{kg}$ endotoxin groups, respectively). A significant difference was noted in the slope of the $20-$ and $30-\mu \mathrm{g} / \mathrm{kg}$ endotoxin groups compared with that of the control $(P<0.001$ for both comparisons) and for $20-\mu \mathrm{g} / \mathrm{kg}$ endotoxin group compared with that of the $30-\mu \mathrm{g} / \mathrm{kg}$ group $(P=0.014)$. The individual values for the SUV slopes are provided in Figure 3. This shows that there is no overlap in the values between groups, with negative values for the control kits and predominantly positive values for the endotoxin-exposed kits. This finding indicates that there is increased retention of ${ }^{11} \mathrm{C}-(R)$-PK11195 over time in the endotoxin-exposed groups compared with that in the control group. This is illustrated in Figure 4, where retention of the tracer is noted in the image representing the last $10 \mathrm{~min}$ of the scan for the $20-$ and $30-\mu \mathrm{g} / \mathrm{kg}$ endotoxin kits, whereas a decline in the tracer concentration over the scanning period is noted in the control rabbit kit. 

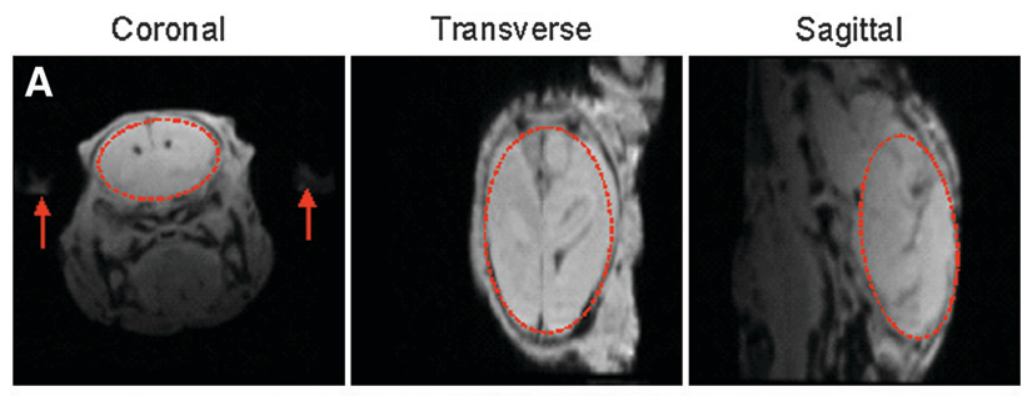

\section{$\mathrm{MBq} / \mathrm{g}$}

1.1
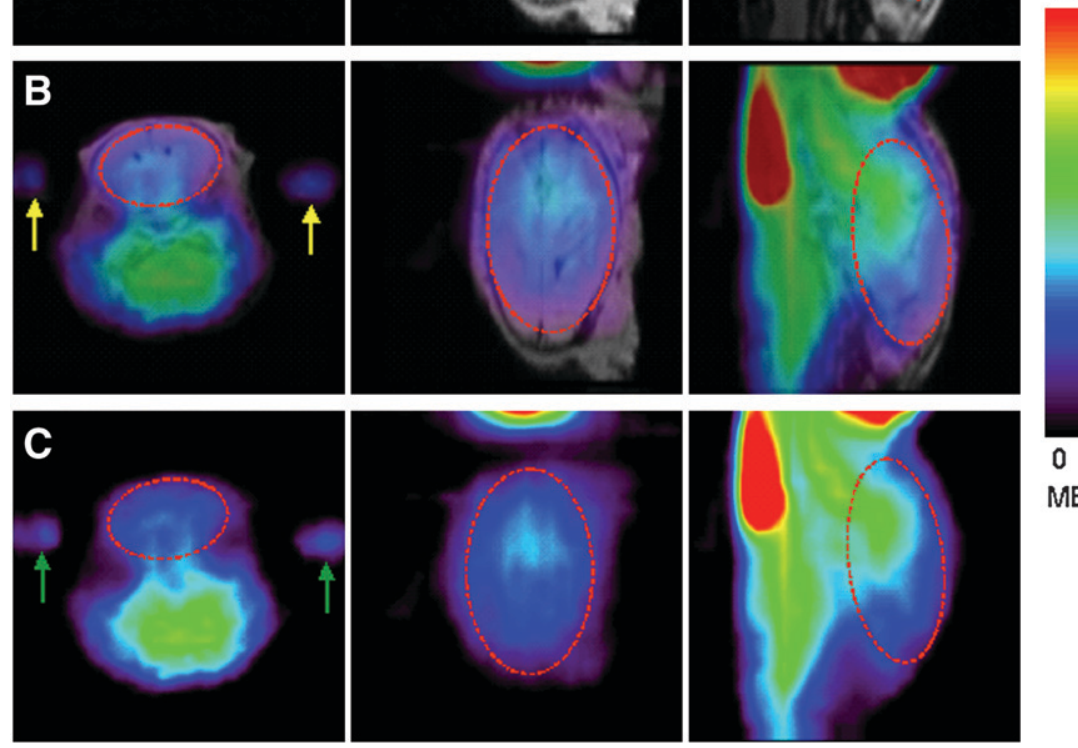

FIGURE 1. Coregistration of $\mathrm{MRI}$ and PET images: a representative newborn rabbit kit (30 $\mu \mathrm{g} / \mathrm{kg}$ of endotoxin) on day 1 of life. (A) T1-weighted MR images. (B) Coregistered images. (C) microPET images. Coregistration was done using markers on a custom-made head holder that is visible on PET and MRI. Red arrows indicate water as marker noted on MR image, and green arrows indicate markers evident on PET scan. Overlap of markers (indicated by yellow arrows) is seen on coregistered images. Because of partial-volume effects, 3D ROls (indicated by orange dashed lines) were drawn for the whole brain and SUV over time was calculated and compared between groups.

There was no significant difference in the mean intercept (SUV estimated at time 0 of scan) of ${ }^{11} \mathrm{C}-(R)-\mathrm{PK} 11195$ retention slope in the brains of the control kits and that of the kits exposed to $20 \mu \mathrm{g} / \mathrm{kg}$ of endotoxin (mean intercept $\pm \mathrm{SE}$ was $0.597 \pm 0.105$ and $0.575 \pm 0.105$ for the control and $20-\mu \mathrm{g} / \mathrm{kg}$ endotoxin groups, respectively; $P=0.887)$. The mean intercept for the $30-\mu \mathrm{g} / \mathrm{kg}$ group was $0.221 \pm 0.105$, which was significantly lower than that of the control $(P=$ $0.032)$ and $20-\mu \mathrm{g} / \mathrm{kg}(P=0.041)$ groups. The lower initial SUV in the $30-\mu \mathrm{g} / \mathrm{kg}$ endotoxin group may be indicative of decreased cerebral blood flow in these kits.

\section{Histology}

The extent of microglial activation was assessed by immunohistochemical staining by determining the morphology and number of microglial cells semiquantitatively. Microglial cells when in a "quiescent" or nonactivated state are typically ramified with multiple branches. With activation the branches become shorter, and eventually the cells completely lose their ramifications and become rounded or amoeboid in shape (Fig. 5). A robust microglial response was noted in the regions of the corpus callosum, especially along the inferior border of the lateral ventricles, around the angle of the lateral ventricles, in the hippocampusincluding dentate gyrus and fimbria hippocampus-and in the internal capsule (Fig. 6). An increase in number and change in morphology of microglial cells from ramified in the control rabbit kits to a more rounded shape with ex- posure to LPS was noted, indicating activation of microglial cells in the kits exposed to endotoxin in utero (Table 1).

\section{DISCUSSION}

Our results demonstrate that exposure to endotoxin in utero leads to a robust microglial response in the newborn rabbit brain detected noninvasively by microPET using the ligand ${ }^{11} \mathrm{C}-(R)-\mathrm{PK} 11195$, which is specific for the PBRs expressed on activated microglial cells. The relationship between maternal infections and fetal brain injury has been demonstrated in both animal experiments and epidemiologic studies. Maternal cytokine-associated inflammatory response appears to be the link in the relationship between infections during pregnancy and the development of cerebral palsy and other neuropsychiatric conditions $(22,23)$. Multiple animal experiments have also confirmed that the type of pathogen appears irrelevant and that, even in the absence of a pathogen, cytokine-releasing treatment during pregnancy produces fetal brain injury $(24,25)$. The endotoxin Escherichia coli LPS was used in this study to create neuroinflammation in the fetal brain, and this has been used in previous animal models of maternal inflammation leading to white matter injury $(26,27)$. We chose gestational day 28 for the endotoxin injection because this time period corresponds to third-trimester pregnancy in humans, when there is a rapid increase in the number of immature oligodendrocytes. In rabbits, the presence of immature 


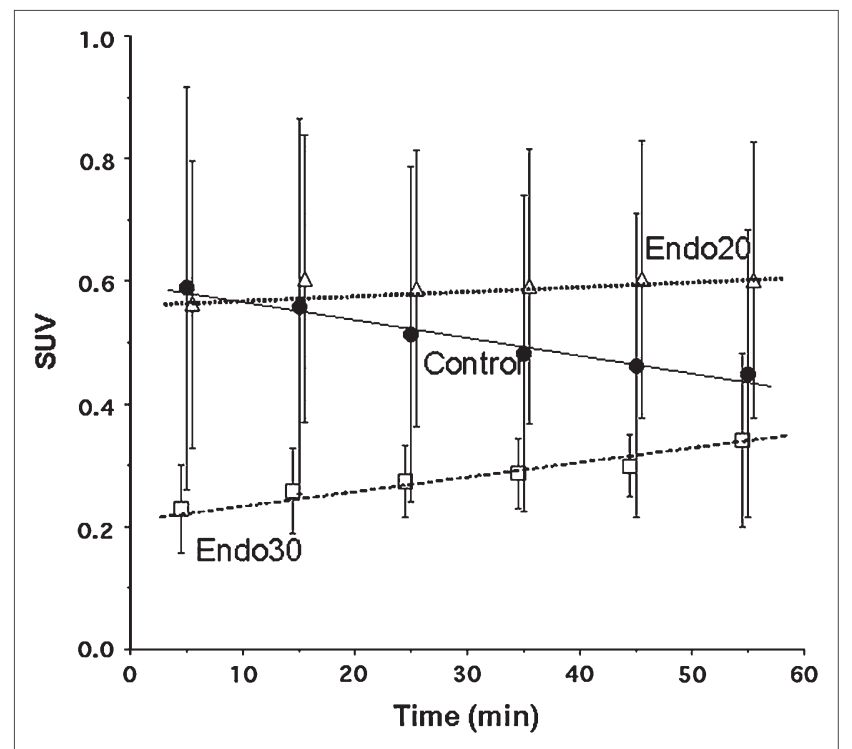

FIGURE 2. Slope of SUV for ${ }^{11} \mathrm{C}-(R)$-PK11195 over time in postnatal day 1 kits. Lines represent slopes for each group estimated by HLM, markers represent mean SUV for all kits in that group for that time point, and error bars indicate SD for mean. Slope was negative for control group (mean slope = $-0.003 \mathrm{~min}^{-1}$ ) and positive for both endotoxin groups (mean slopes $=+0.0006 \mathrm{~min}^{-1}$ for $20-\mu \mathrm{g} / \mathrm{kg}$ endotoxin $\mathrm{kits}$ [Endo20] and $+0.002 \mathrm{~min}^{-1}$ for $30-\mu \mathrm{g} / \mathrm{kg}$ endotoxin kits [Endo30]), indicating retention of tracer in brain due to specific binding of the tracer to peripheral benzodiazepine binding sites in newborn rabbits exposed to endotoxin in utero. Upregulation of binding sites suggests that there is increased microglial activation in brain of kits exposed to endotoxin in utero.

oligodendrocytes starts around gestational day 25, with a rapid increase in its number until the onset of myelination on postnatal day 5, reaching a plateau on postnatal day 11 (28). Myelination begins in the internal capsule on postnatal day 5 and in the corpus callosum on postnatal day 11 . The development of immature oligodendrocytes and the functional maturation of axons, especially in the areas around the corpus callosum and internal capsule, occur in

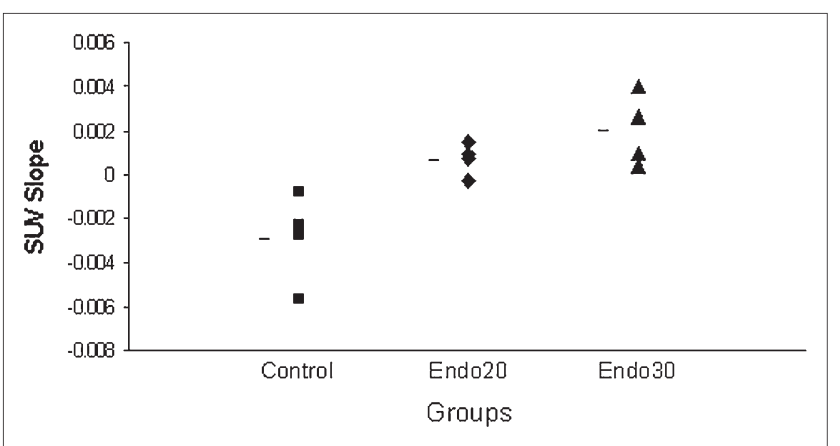

FIGURE 3. SUV slope values for each group. Individual slopes for each kit in control, $20 \mu \mathrm{g} / \mathrm{kg}$ of endotoxin (Endo20), and 30 $\mu \mathrm{g} / \mathrm{kg}$ of endotoxin (Endo30) groups are depicted. Mean slope for each group is represented as horizontal line. There is no overlap in slope values between control and endotoxin groups. the time period around birth in rabbits (28). In humans, immature oligodendrocytes increase rapidly in numbers in the third trimester; myelination begins around term and is most rapid in the first year of life (29). Hence, myelination in rabbits is similar to that in humans in that it is starts in the perinatal period. Because chorioamnionitis and maternal intrauterine inflammation are known to be associated epidemiologically with cerebral palsy both in term and in preterm infants, a time point closer to term-when oligodendrocyte maturation is still ongoing-was chosen for endotoxin injection.

The peripheral benzodiazepine binding sites or receptors are multimeric protein complexes (30) that are most commonly located in activated microglia in the brain and have been used as a sensitive marker to visualize and measure microglial cell activation associated with various neuroinflammatory disorders $(12,13)$. Though the exact function of the PBR has not been clearly elucidated, it appears to be involved with physiologic processes such as cell proliferation, steroidogenesis, and immunomodulation (30). Because of the tight correlation between the expression of peripheral benzodiazepine binding sites and the presence of activated glial cells, which has been demonstrated in several studies, the detection of this receptor has been developed as a marker for the presence of numerous brain diseases. The activation of microglial cells is typically localized to the site of the injured neuron with extension along the anterograde or retrograde axonal pathway, unlike astrogliosis that can spread diffusely in response to injury. This characteristic response would help localize the site and distribution of injury more accurately when imaging activated microglia, providing information about the temporal and spatial progression of various neuroinflammatory disorders.

In this study we demonstrated an increase in the retention of ${ }^{11} \mathrm{C}-(R)-\mathrm{PK} 11195$, as indicated by a change in the slope of the tracer SUV time-activity curve in the newborn rabbit brain after in utero exposure to endotoxin. Because of the small size of the newborn rabbit kits, tracer kinetic modeling with repeated arterial blood sampling for measurement of the arterial input function was not feasible. Hence, in this model, the slope of the SUV time-activity curve between 5 and $65 \mathrm{~min}$ after injection was used as a semiquantitative assessment of the tracer retention as an indicator of specific binding of the ligand to PBRs in activated microglial cells in the brain. This is confirmed by our immunohistochemical results, which clearly demonstrate an increase in the number, and a change in morphology, of the microglial cells in specific regions of the newborn rabbit brain exposed to endotoxin in utero. These results are in accordance with studies using ${ }^{3} \mathrm{H}-(R)-$ PK11195 autoradiography in experimental encephalomyelitis (12) and ${ }^{11} \mathrm{C}-(R)-\mathrm{PK} 11195$ PET in patients with multiple sclerosis or cerebrovascular disease, which have predominantly shown increased binding of the ligand to activated microglia and infiltrating macrophages $(13,17)$. 

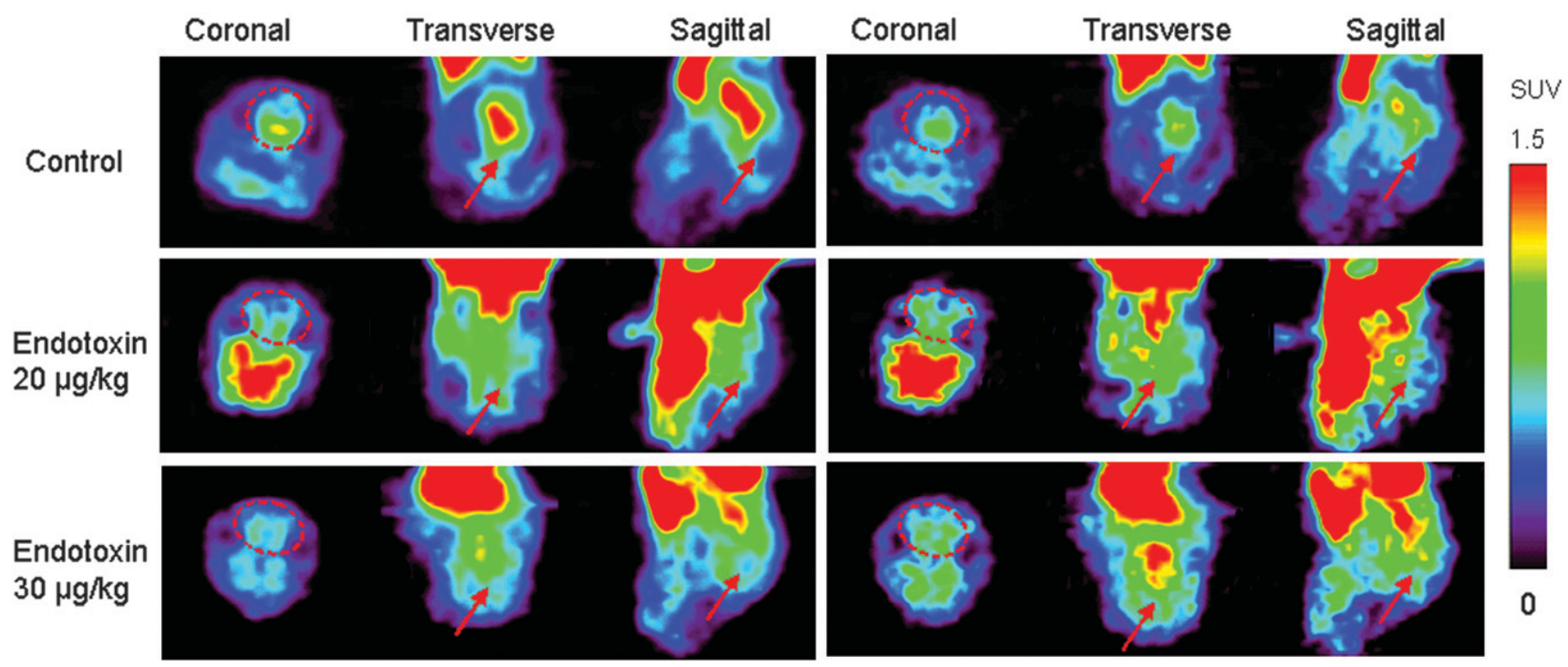

FIGURE 4. PET images of ${ }^{11} \mathrm{C}-(R)-\mathrm{PK} 11195$ uptake in brain in first 10 min of scan (frame 1) and in last 10 min of scan (frame 6 ) on day 1 of life. Red circle indicates ROI involving whole brain that was drawn to measure uptake in that region. Red arrows indicate region of interventricular zone at level of hippocampus and dentate gyrus. Activity in brain decreases over time for saline-injected control kits, whereas it increases over time for newborn rabbit kits exposed to endotoxin in utero, indicating specific binding of tracer to activated microglial cells in endotoxin-exposed kits. Image intensity is adjusted for dose injected and weight of animal and the SUV scale provided.

Similarly, Chen et al., using a mouse model of cuprizoneinduced demyelination, have shown that increased ${ }^{3} \mathrm{H}-$ $(R)$-PK11195 binding to PBRs was associated with glial activation and also correlated with the degree of demyelination (31).

In our study, the initial SUVs (indicated as estimated mean intercept of the slopes for each group) were similar for the control and the $20-\mu \mathrm{g} / \mathrm{kg}$ endotoxin groups. However, a lower initial SUV for ${ }^{11} \mathrm{C}-(\mathrm{R})-\mathrm{PK} 11195$ was noted in the kits exposed to $30 \mu \mathrm{g} / \mathrm{kg}$ of endotoxin compared with that of the control and $20-\mu \mathrm{g} / \mathrm{kg}$ endotoxin kits. This may be due to decreased cerebral perfusion in the kits exposed to higher doses of endotoxin. Thus, ischemia may also be responsible for microglial activation in the kits exposed to $30 \mu \mathrm{g} / \mathrm{kg}$ endotoxin, whereas in the kits exposed to the lower dose of endotoxin in utero, microglial activation appears to be predominantly due to inflammation induced by endotoxin exposure.

Microglial cells constitute about $10 \%-20 \%$ of the cells in the adult brain and are activated by pathologic stimuli such as trauma, infection, inflammation, and ischemia. As a result of these stimuli, they change their morphology from a ramified to an amoeboid form (32). In vitro studies have showed that microglial cells retract their processes after exposure to LPS and interferon- $\gamma$ (33). Cell culture studies have demonstrated their rapid transformation from ramified to amoeboid cells within $2 \mathrm{~h}$ of exposure to adenosine triphosphate (ATP), and the removal of ATP was associated with reversal of the cells to their resting ramified state (34). The activated microglial cells rapidly respond as the first line of defense in the brain in an attempt to wall off the injury. This is noted to occur within minutes to a few hours after the original injury (35). These microglial cells that have accumulated at the site of injury subsequently proliferate and significantly increase in numbers, with a peak in their mitotic activity noted around $3 \mathrm{~d}$ after the initial injury $(32,36)$. In the absence of any further injury, these cells return to their ramified, "resting" state but in the presence of ongoing neuronal loss they remain activated and phagocytose the cell debris. Microglial cells may also be
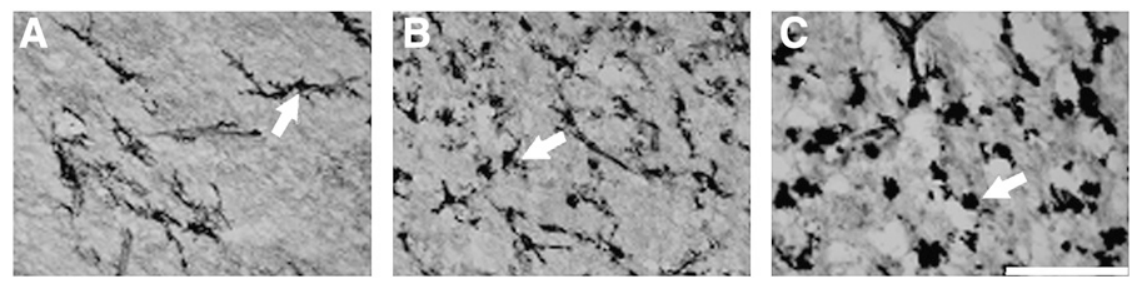

FIGURE 5. Morphology of microglial cells using tomato lectin staining. (A) In control group, cells are highly ramified (arrow). (B and C) In endotoxin-treated group, cells had shorter and thicker processes. In this latter group, cells are bushy $(\mathrm{B}$, arrow), becoming amoeboid and round (C, arrow). Increase in density of microglial cells is observed in endotoxin-treated group compared with that of control group. Scale bar $=100 \mu \mathrm{m}$. 


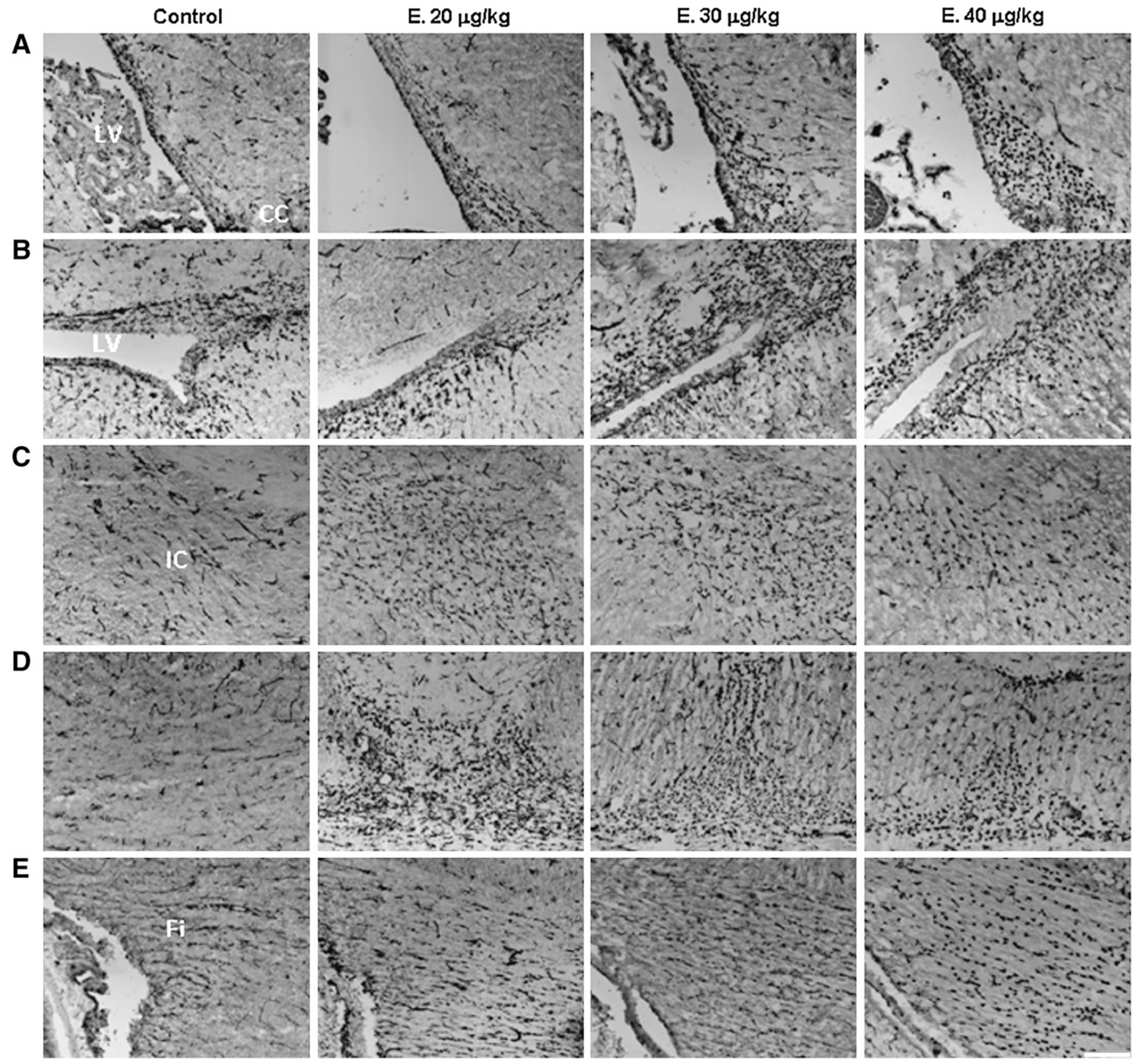

FIGURE 6. Localization of microglial cells and morphology using tomato lectin staining in control kits and in kits exposed to 20 , 30 , and $40 \mu \mathrm{g} / \mathrm{kg}$ of endotoxin. Microglial cells are seen in corpus callosum, along inferior border of lateral ventricle (A), around angle of lateral ventricle (B), in area of internal capsule (C), between 2 fields of hippocampus (D), and in fimbria hippocampus (E). Increase in density of microglial cells is observed in endotoxin-treated groups compared with control group. In all of these regions, microglial cells are ramified in control group and become less ramified/bushy, amoeboid, and round in endotoxin-treated groups. E. = endotoxin; LV = lateral ventricle; $\mathrm{CC}=$ corpus callosum; IC = internal capsule; Fi = fimbria hippocampus. Scale bar $=200$ $\mu \mathrm{m}$.

activated by proinflammatory cytokines $(5,6)$, which are produced by the mother or the fetus in response to intrauterine infection (22). In the presence of infection or inflammation, there is also upregulation of several cell adhesion markers along with secretion of proinflammatory mediators, generation of reactive oxygen species, and peroxynitrites that may lead to further neuronal damage (8).

Activated microglial cells are prominent during fetal life as part of normal embryonic development (37) and are found in increased density in regions of white matter tracts in late gestation with a decrease in density around term, but maintaining their amoeboid morphology even in early infancy (38). Hence, any brain injury around term will involve the activation of microglial cells that are normally present abundantly in white matter tracts, making this a vulnerable period for oligodendrocyte and myelin damage. Exposure to increasing doses of endotoxin in utero in our model was associated with a change in morphology of the microglial cells from a ramified to an amoeboid form and an increase in the number of cells. Detection of activated 
TABLE 1

Location of Microglial Cells and Morphology

\begin{tabular}{|c|c|c|c|c|}
\hline \multirow[b]{2}{*}{ Site } & \multirow[b]{2}{*}{ Control group } & \multicolumn{3}{|c|}{ Endotoxin group } \\
\hline & & $20 \mu \mathrm{g} / \mathrm{kg}$ & $30 \mu \mathrm{g} / \mathrm{kg}$ & $40 \mu \mathrm{g} / \mathrm{kg}$ \\
\hline Frontal cortex & - & - & - & - \\
\hline Corpus callosum: along inferior border of lateral ventricle & $\begin{array}{l} \pm \\
\mathrm{Rm}\end{array}$ & $\begin{array}{l}+ \\
\mathrm{Bu}\end{array}$ & $\begin{array}{l}++ \\
\mathrm{Bu}, \mathrm{Ro}, \mathrm{Am}\end{array}$ & $\begin{array}{l}+++ \\
\mathrm{Ro}, \mathrm{Am}\end{array}$ \\
\hline Internal capsule & $\begin{array}{l}+ \\
\mathrm{Rm}\end{array}$ & $\begin{array}{l}+++ \\
\mathrm{Bu}\end{array}$ & $\begin{array}{l}+++ \\
\mathrm{Bu}, \mathrm{Ro}, \mathrm{Am}\end{array}$ & $\begin{array}{l}++ \\
\mathrm{Ro}, \mathrm{Am}\end{array}$ \\
\hline External capsule & - & - & - & - \\
\hline Around angle of lateral ventricle (at level of thalamus) & $\begin{array}{l}++ \\
\mathrm{Rm}\end{array}$ & $\begin{array}{l}++ \\
\mathrm{Bu}\end{array}$ & $\begin{array}{l}+++ \\
\mathrm{Bu}, \mathrm{Ro}, \mathrm{Am}\end{array}$ & $\begin{array}{l}+++ \\
\mathrm{Bu}, \mathrm{Ro}, \mathrm{Am}\end{array}$ \\
\hline Thalamus & - & - & - & - \\
\hline $\begin{array}{l}\text { Parietal cortex } \\
\text { Hippocampus }\end{array}$ & - & - & - & - \\
\hline Between 2 fields of hippocampus & $\begin{array}{l}+ \\
\mathrm{Rm}\end{array}$ & $\begin{array}{l}+++ \\
\mathrm{Bu}\end{array}$ & $\begin{array}{l}+++ \\
\text { Ro, Am }\end{array}$ & $\begin{array}{l}+++ \\
\text { Ro, Am }\end{array}$ \\
\hline Dentate gyrus & $\begin{array}{l}+ \\
\mathrm{Rm}\end{array}$ & $\begin{array}{l}+ \\
\mathrm{Bu}\end{array}$ & $\begin{array}{l}+ \\
\mathrm{Ro}, \mathrm{Am}\end{array}$ & $\begin{array}{l}+++ \\
\mathrm{Ro}, \mathrm{Am}\end{array}$ \\
\hline Fimbria hippocampus & $\begin{array}{l}+ \\
\mathrm{Rm}\end{array}$ & $\begin{array}{l}++ \\
\mathrm{Rm}\end{array}$ & $\begin{array}{l}++ \\
\mathrm{Bu}, \mathrm{Ro}, \mathrm{Am}\end{array}$ & $\begin{array}{l}+++ \\
\text { Ro, Am }\end{array}$ \\
\hline
\end{tabular}

$-=$ no microglial cells; $\pm=$ rare microglial cells; $+=$ few microglial cells; $++=$ some microglial cells; $+++=$ many microglial cells; $\mathrm{Rm}=$ ramified cells; $\mathrm{Bu}=$ bushy cells; $\mathrm{Ro}=$ round cells; $\mathrm{Am}=$ amoeboid cells.

microglial cells in vivo can provide valuable information about the degree of injury sustained in the perinatal period. Neonates born to mothers with chorioamnionitis either diagnosed clinically or by placental histopathology, can be screened by PET with ${ }^{11} \mathrm{C}-(R)$-PK11195 for the presence of activated microglial cells as an indicator of neuroinflammation and, hence, possible predictor for the development of brain injury secondary to intrauterine infection. Though large radiation exposures in the neonatal period is a matter for concern, with regard to the radiation dose, ${ }^{11} \mathrm{C}-(R)$ PK11195 scans in neonates could be accomplished using effective doses approximately equal to the yearly background radiation exposure and less than or equal to the exposure of a clinical CT scan of the head. Although there is evidence that children are more sensitive to radiation than adults at higher radiation doses, at low doses such as those associated with PET and other medical diagnostic imaging, there is no evidence for adverse effects in either children or adults (39). Given the serious lifelong, debilitating consequences of the development of cerebral palsy in neonates from perinatal insults, early detection of neuroinflammation may result in a high potential benefit-to-risk ratio. Judicious use of PET in neonates and infants may be very helpful in understanding the mechanisms of brain injury and neuroinflammation in the developing brain, as well as in early identification of neonates exposed to intrauterine infection in need of treatment for neuroinflammation.

\section{CONCLUSION}

Our data suggest that PET with the ligand ${ }^{11} \mathrm{C}-(R)-$ PK11195 can be used in the neonatal period as a sensitive tool for the early detection of neuropathologic changes that result from perinatal insults. Because transformation of microglia to an activated state is linked to progression of the injury, imaging of peripheral benzodiazepine binding sites may be used clinically to determine the risk of developing cerebral palsy and other neuropsychiatric conditions and to follow disease progression and response to therapy. Further studies must be done to determine the time course and progression of microglial activation and correlate it with functional deficits in the neonate.

\section{ACKNOWLEDGMENTS}

This research was supported (in part) by the Intramural Research Program of the National Institute of Child Health and Human Development, NIH, DHHS, and the Pediatric Critical Care Scientist Development Program of the NICHD. We thank Xin Lu for his technical support and Dr. Elizabeth Dawe for her help with the animal model.

\section{REFERENCES}

1. Dammann O, Leviton A. Maternal intrauterine infection, cytokines, and brain damage in the preterm newborn. Pediatr Res. 1997;42:1-8.

2. Grether JK, Nelson KB. Maternal infection and cerebral palsy in infants of normal birth weight. JAMA. 1997;278:207-211.

3. Wu YW, Colford JM Jr. Chorioamnionitis as a risk factor for cerebral palsy: a meta-analysis. JAMA. 2000;284:1417-1424.

4. Haynes RL, Folkerth RD, Keefe RJ, et al. Nitrosative and oxidative injury to premyelinating oligodendrocytes in periventricular leukomalacia. J Neuropathol Exp Neurol. 2003;62:441-450.

5. Takeuchi H, Jin S, Wang J, et al. Tumor necrosis factor-alpha induces neurotoxicity via glutamate release from hemichannels of activated microglia in an autocrine manner. J Biol Chem. 2006;281:21362-21368.

6. Hailer NP, Vogt C, Korf HW, Dehghani F. Interleukin-1beta exacerbates and interleukin-1 receptor antagonist attenuates neuronal injury and microglial activation after excitotoxic damage in organotypic hippocampal slice cultures. Eur J Neurosci. 2005;21:2347-2360. 
7. Lehnardt S, Lachance C, Patrizi S, et al. The toll-like receptor TLR4 is necessary for lipopolysaccharide-induced oligodendrocyte injury in the CNS. J Neurosci. 2002;22:2478-2486.

8. Li J, Baud O, Vartanian T, Volpe JJ, Rosenberg PA. Peroxynitrite generated by inducible nitric oxide synthase and NADPH oxidase mediates microglial toxicity to oligodendrocytes. Proc Natl Acad Sci U S A. 2005;102:9936-9941.

9. Espey MG, Chernyshev ON, Reinhard JF Jr, Namboodiri MA, Colton CA. Activated human microglia produce the excitotoxin quinolic acid. Neuroreport. 1997;8:431-434.

10. Banati RB, Myers R, Kreutzberg GW. PK ('peripheral benzodiazepine')-binding sites in the CNS indicate early and discrete brain lesions: microautoradiographic detection of [ $\left.{ }^{3} \mathrm{H}\right] \mathrm{PK} 11195$ binding to activated microglia. J Neurocytol. 1997;26: 77-82.

11. Shah F, Hume SP, Pike VW, Ashworth S, McDermott J. Synthesis of the enantiomers of [N-methyl- $\left.{ }^{11} \mathrm{C}\right] \mathrm{PK} 11195$ and comparison of their behaviours as radioligands for PK binding sites in rats. Nucl Med Biol. 1994;2:573-581.

12. Vowinckel E, Reutens D, Becher B, et al. PK11195 binding to the peripheral benzodiazepine receptor as a marker of microglia activation in multiple sclerosis and experimental autoimmune encephalomyelitis. J Neurosci Res. 1997;50:345-353.

13. Banati RB, Newcombe J, Gunn RN, et al. The peripheral benzodiazepine binding site in the brain in multiple sclerosis: quantitative in vivo imaging of microglia as a measure of disease activity. Brain. 2000;123:2321-2337.

14. Turner MR, Cagnin A, Turkheimer FE, et al. Evidence of widespread cerebral microglial activation in amyotrophic lateral sclerosis: an $\left[{ }^{11} \mathrm{C}\right](\mathrm{R})-\mathrm{PK} 11195$ positron emission tomography study. Neurobiol Dis. 2004;15:601-609.

15. Ouchi Y, Yoshikawa E, Sekine Y, et al. Microglial activation and dopamine terminal loss in early Parkinson's disease. Ann Neurol. 2005;57:168-175.

16. Pappata S, Levasseur M, Gunn RN, et al. Thalamic microglial activation in ischemic stroke detected in vivo by PET and $\left[{ }^{11} \mathrm{C}\right] \mathrm{PK} 1195$. Neurology. 2000;55: 1052-1054.

17. Gerhard A, Schwarz J, Myers R, Wise R, Banati RB. Evolution of microglial activation in patients after ischemic stroke: a $\left[{ }^{11} \mathrm{C}\right](\mathrm{R})-\mathrm{PK} 11195$ PET study. Neuroimage. 2005;24:591-595.

18. Chakraborty PK, Maiti DK, Mangner TJ, et al. High yield and semi-automated synthesis procedure of (R)-[ $\left[{ }^{11} \mathrm{C}\right] \mathrm{PK} 11195$ [abstract]. J Nucl Med. 2006; 47(suppl):522P.

19. Fueger BJ, Czernin J, Hildebrandt I, et al. Impact of animal handling on the results of ${ }^{18}$ F-FDG PET studies in mice. J Nucl Med. 2006;47:999-1006.

20. Pietrzyk U, Herholz K, Heiss WD. Three-dimensional alignment of functional and morphological tomograms. J Comput Assist Tomogr. 1990;14:51-59.

21. Siegel S, Castellan NJ Jr. Nonparametric Statistics for the Behavioral Sciences. New York, NY: McGraw-Hill. 2nd ed; 1988.

22. Yoon BH, Jun JK, Romero R, et al. Amniotic fluid inflammatory cytokines (interleukin-6, interleukin-1beta, and tumor necrosis factor-alpha), neonatal brain white matter lesions, and cerebral palsy. Am J Obstet Gynecol. 1997;177:19-26.
23. Meyer U, Feldon J, Schedlowski M, Yee BK. Towards an immuno-precipitated neurodevelopmental animal model of schizophrenia. Neurosci Behav Rev. 2005; 29:913-947.

24. Bell MJ, Hallenbeck JM, Gallo V. Determining the fetal inflammatory response in an experimental model of intrauterine inflammation in rats. Pediatr Res. 2004; 56:541-546.

25. Shi L, Tun N, Patterson PH. Maternal influenza infection is likely to alter fetal brain development indirectly: the virus is not detected in the fetus. Int $J$ Dev Neurosci. 2005;23:299-305.

26. Cai Z, Pan ZL, Pang Y, Evans OB, Rhodes PG. Cytokine induction in fetal rat brains and brain injury in neonatal rats after maternal lipopolysaccharide administration. Pediatr Res. 2000;47:64-72.

27. Bell MJ, Hallenbeck JM. Effects of intrauterine inflammation on developing rat brain. J Neurosci Res. 2002;70:570-579.

28. Drobyshevsky A, Song SK, Gamkrelidze G, et al. Developmental changes in diffusion anisotropy coincide with immature oligodendrocyte progression and maturation of compound action potential. J Neurosci. 2005;25:5988-5997.

29. de Graaf-Peters VB, Hadders-Algra M. Ontogeny of the human central nervous system: What is happening when? Early Hum Dev. 2006;82:257-266.

30. Papadopoulos V, Baraldi M, Guilarte TR, et al. Translocator protein (18kDa): new nomenclature for the peripheral-type benzodiazepine receptor based on its structure and molecular function. Trends Pharmacol Sci. 2006;27:402-409.

31. Chen MK, Baidoo K, Verina T, Guilarte TR. Peripheral benzodiazepine receptor imaging in CNS demyelination: functional implications of anatomical and cellular localization. Brain. 2004;127:1379-1392.

32. Raivich G, Bohatschek M, Kloss CU, Werner A, Jones LL, Kreutzberg GW. Neuroglial activation repertoire in the injured brain: graded response, molecular mechanisms and cues to physiological function. Brain Res Brain Res Rev. 1999;30:77-105.

33. Giulian D, Li J, Bartel S, Broker J, Li X, Kirkpatrick JB. Cell surface morphology identifies microglia as a distinct class of mononuclear phagocyte. J Neurosci. 1995; 15:7712-7726.

34. Xiang Z, Chen M, Ping J, et al. Microglial morphology and its transformation after challenge by extracellular ATP in vitro. J Neurosci Res. 2006;83:91-101.

35. Davalos D, Grutzendler J, Yang G, et al. ATP mediates rapid microglial response to local brain injury in vivo. Nat Neurosci. 2005;8:752-758.

36. Herber DL, Maloney JL, Roth LM, et al. Diverse microglial responses after intrahippocampal administration of lipopolysaccharide. Glia. 2006;53:382-391.

37. Chugani DC, Kedersha N, Rome LH. Vault immunofluorescence in the brain: new insights regarding the origin of microglia. J Neurosci. 1991;11:256-268.

38. Billiards SS, Haynes RL, Folkerth RD, et al. Development of microglia in the cerebral white matter of the human fetus and infant. J Comp Neurol. 2006;497: 199-208.

39. Preston R. Children as a sensitive subpopulation for the risk assessment process. Toxicol Appl Pharmacol. 2004;199:132-141. 